\title{
Examining injury risk and pain perception in runners using minimalist footwear
}

\author{
Michael Ryan, ${ }^{1}$ Maha Elashi, ${ }^{2}$ Richard Newsham-West, ${ }^{1}$ Jack Taunton ${ }^{2}$
}

- Additional material is published online only. To view please visit the journal online (http://dx.doi.org/10.1136/ bjsports-2012-092061).

${ }^{1}$ Centre for Musculoskeletal Research, Griffith University, Southport, Queensland, Australia

${ }^{2}$ Division of Sports Medicine, Faculty of Medicine, University of British Columbia, Vancouver British Columbia, Canada

\section{Correspondence to} Dr Michael Ryan, Centre for Musculoskeletal Research, Gold Coast Campus,

Griffith University, Southport, Queensland 42222, Australia; mryan76@gmail.com

Accepted 2 December 2013

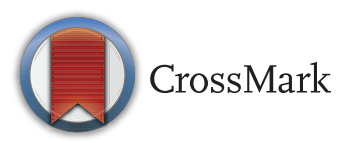

To cite: Ryan $\mathrm{M}$, Elashi $\mathrm{M}$ Newsham-West R, et al. Br J Sports Med Published Online First: [please include Day Month Year] doi:10.1136/ bjsports-2012-092061

\section{ABSTRACT}

Background This study examines the effect of progressive increases in footwear minimalism on injury incidence and pain perception in recreational runners. Methods One hundred and three runners with neutral or mild pronation were randomly assigned a neutral (Nike Pegasus 28), partial minimalist (Nike Free 3.0 V2) or full minimalist shoe (Vibram 5-Finger Bikila). Runners underwent baseline testing to record training and injury history, as well as selected anthropometric measurements, before starting a 12-week training programme in preparation for a $10 \mathrm{~km}$ event. Outcome measures included number of injury events, Foot and Ankle Disability (FADI) scores and visual analogue scale pain rating scales for regional and overall pain with running.

Results 99 runners were included in final analysis with 23 injuries reported; the neutral shoe reporting the fewest injuries (4) and the partial minimalist shoe (12) the most. The partial minimalist shoe reported a significantly higher rate of injury incidence throughout the 12-week period. Runners in the full minimalist group reported greater shin and calf pain.

Conclusions Running in minimalist footwear appears to increase the likelihood of experiencing an injury, with full minimalist designs specifically increasing pain at the shin and calf. Clinicians should exercise caution when recommending minimalist footwear to runners otherwise new to this footwear category who are preparing for a $10 \mathrm{~km}$ event.

\section{INTRODUCTION}

In recent years, the running footwear industry has undergone a transformation based in part on the theoretical advantages of barefoot or minimalist running. These advantages have been outlined in evolutionary terms ${ }^{1}{ }^{2}$ with biomechanical consequences reducing impact forces and/or internal joint loads leading to the potential to lower injury incidence rates. ${ }^{3}{ }^{4}$ Laboratory studies comparing barefoot to $\operatorname{shod}^{5} 6$ and to footwear designed to mimic a barefoot condition (Vibram 5-Fingers) ${ }^{7}$ report significant changes to running mechanics. Specifically, running barefoot typically results in a higher step rate, more plantarflexed foot to ground angle at touch down and reduced maximum knee and hip flexion during midstance. ${ }^{5}{ }^{6}$ From a clinical perspective, the lower resulting internal knee and hip extension, and hip abduction moments indeed suggest that footwear minimalism (running barefoot) may be protective for injury. 89

There is no consensus of what defines a minimalist running shoe. Broadly speaking, minimalist footwear may be defined as incorporating design aspects which aim to reduce mechanical and/or sensory interference between the shoe and foot. ${ }^{10}$ In manufacturing terms running shoe minimalism is achieved by a reduction in one or more of the following: midsole thickness, forefoot to heel elevation, heel counter stiffness and midsole stability elements. Conventionally, running shoes have a stack-height (thickness of material between the ground and foot including outsole, midsole and insole) of greater than $30 \mathrm{~mm}$ with a $10-12 \mathrm{~mm}$ elevated heel. ${ }^{11}$ Minimalist footwear as a category has varied design characteristics in terms of sole construction, but will be defined for the purpose of this study as a footwear model having less than $30 \mathrm{~mm}$ of rearfoot stack-height combined with a heel lift of less than $10 \mathrm{~mm}$.

Two models of minimalist running footwear have been investigated previously, Nike's Free V.5.0 $0^{12}$ and Vibram's 5-Finger Bikila. ${ }^{7}$ These respective footwear models have demonstrated significant changes in muscle physiology and stride dynamics that may result in reductions to a runner's risk of injury. Despite the plausible assertion that wearing certain models of minimalist footwear may be protective for injury in certain individuals, no epidemiological investigation has investigated how minimalism in footwear might translate into changes in injury risk in any running population. The objective of the present study; therefore, was to prospectively determine the effect of two different minimalist footwear models, and one conventional model, on injury incidence and pain in a population of recreational runners. Based on prior biomechanical investigations with the use of selected minimalist footwear models the following hypotheses are formulated: (1) Increasing footwear minimalism will modify injury risk and (2) Increasing footwear minimalism will also modify overall self-reported pain and functional disability.

\section{Methodology}

Participants

A prospective randomised clinical trial (Registry ClinicalTrials.gov: NCT01334346) was conducted in Vancouver, Canada, on the clinical effects of three different running footwear models. The intervention investigated was increasing degree of footwear minimalism, specifically decreasing mid-sole thickness, decreasing heel height and reduced stiffness of the mid-sole underneath the forefoot. The control group in this study included individuals receiving a conventional neutral supportive running shoe (see online supplementary table S1). Ethical approval for this study was obtained from the Clinical Research Ethics Board at the University of British Columbia and all participants were given full informed consent before taking part. 
Participants were recruited primarily through a newspaper advertisement and word of mouth. Participants recruited were adults (men and women) between the ages of 19 and 50 who have a minimum of 5 years' running experience, been running on a regular basis (minimum once per week) over the past 6 months, were able to run for 60 min continuously and could tolerate $20-40 \mathrm{~km} /$ week training programme, and had no running-related injury requiring a stoppage of training for 2 weeks or more in the past 6 months. Individuals were excluded if they had a history of surgery to their plantar fascia or Achilles tendon, had a diagnosis of osteoarthritis or other degenerative musculoskeletal disorder affecting the lower extremity or are currently taking analgesic medication. Individuals who already use minimalist running footwear were also excluded.

\section{Baseline assessment}

During the baseline assessment, a detailed training and injury history was taken which included information on running experience, previous $10 \mathrm{~km}$, half-marathon and marathon best finishing time (if available), overall number and type of general musculoskeletal and/or running injuries and previous running shoe make and model. Each participant's foot posture index (FPI) was documented and only individuals with feet categorised as 'neutral', 'supinated' or 'pronated' according to the guidelines by Redmond et $a l^{13}{ }^{14}$ were included in this study; foot postures at the extremes listed as 'highly pronated' or 'highly supinated' were excluded. The FPI has undergone clinometric validation and reliability testing, and has been incorporated in several clinical investigations. ${ }^{15} 16$ The following anthropometric and alignment variables were recorded to test homogeneity between groups of potential confounders based on existing direct or indirect evidence provided in the literature: height, weight, BMI, passive Hallux dorsiflexion range of motion (ROM), quadriceps or (Q) angle and knee alignment. Hallux ROM was assessed non-weightbearing using a hand-held goniometer measuring the angle from the line joining the centre of the first metatarsophalangeal (MTP) joint to the centre of the distal first phalanx to the line joining the centre of the first MTP joint to the centre of the calcaneus when the Hallux was passively dorsiflexed to endpoint. ${ }^{17} \mathrm{Q}$-angle was measured with the participant lying supine and reporting the angle formed from the line joining the anterior superior iliac spine (ASIS) to the centre of the patella to the line joining the centre of the patella to the centre of the tibial tuberosity. ${ }^{18}$ Knee alignment was assessed with the participant standing with their lower limbs together, then using a tape to measure the distance between the medial malleoli in a valgus alignment or the distance between the medial femoral epicondyles in a varus alignment. ${ }^{19}$ All assessments were performed by the same observer (MR) with over 10 years' experience in anthropometric and gait assessments. For concealed group allocation, participants were then randomly assigned by a different author (ME) (via a computer-generated block randomisation scheme-block size 8) to one of the three footwear conditions: neutral footwear (Nike Pegasus), partial-minimalist footwear (Nike Free 3.0 V.2) or fullminimalist footwear (Vibram 5-Finger Bikila).

\section{Training programme}

Following a 1-week break-in period to their assigned footwear, participants began a 12 -week run training programme developed by the authors targeting a $10 \mathrm{~km}$ run held in Vancouver, British Columbia in November, 2011. The programme followed a gradual increase in total running minutes from $160 \mathrm{~min}$ the first week to a peak of $215 \mathrm{~min}$ in week 10 before a 2-week taper. Participants did not always run in their assigned footwear, rather had a gradual increase in exposure as a percentage of their total weekly running time starting at $10 \mathrm{~min}(19 \%$ of volume) in week 1 to $115 \mathrm{~min}(58 \%)$ in week 12 . If a participant felt that repeated use of a shoe was significantly contributing to pain anywhere in the lower extremity, they were given the option of withdrawing from the study.

The programme incorporated three to four run workouts a week, with a longer group run on the weekend and interval training during the middle of the week. The 2-3-weekday workouts were based on time, while the long run on the weekend was based on distance, in order to accommodate different training paces but ensuring adequate preparation for the $10 \mathrm{~km}$ event. The group run was implemented to provide the participants with a positive training environment, and an opportunity to ask questions on their training or other aspects of running in order to optimise compliance. The distance covered during any workout would vary depending on running pace, but it was estimated that the weekly volume started at approximately $15 \mathrm{~km}$ and increased to $30-40 \mathrm{~km}$ at the peak of the programme. There was one lower volume week (week 5) that assigned $60 \%$ of the previous weeks' run volume.

\section{Outcome measures}

Outcome variables of interest in this study included: (1) number of injury events defined as three consecutive missed run workouts secondary to running-related pain; (2) the FADI; (3) overall $100 \mathrm{~mm}$ visual analogue scale (VAS) items for pain associated with running; (4) and anatomical location specific $100 \mathrm{~mm}$ VAS pain associated with running at the foot, lower leg, knee, groin/pelvis and lower back. Outcome variables were assessed at baseline, at the 2-week, 4-week and 8-week point and for the primary comparison at the conclusion of the running programme at week 12. Compliance was monitored with a weekly online survey.

\section{Analysis}

All data were entered into a personal computer and analysed using PASW statistical software (V.17.0.2, SPSS Inc, Chicago, Illinois, USA). An intention to treat analysis approach was used in comparing the effects of one shoe to the others. A last value carried forward strategy was used for missing data (due to withdrawals resulting from injury and/or forgoing wearing assigned footwear) in the cases where participant's had reported a minimum of one follow-up outcome score. ${ }^{20}$ Participants who reported fewer than one follow-up data point were considered drop-outs and their data were omitted for the purpose of analysis. In certain circumstances omitting cases may be justified when the baseline values are not significantly different than overall group, the drop-outs occurred early in the study before a follow-up could be performed and imputation of the data may compromise the overall analysis. ${ }^{21}$

Calculating statistical power in this study was based on the primary outcome measure of participant-reported running-related pain. A 20\% difference in the VAS pain scores across groups with musculoskeletal pain is considered to be clinically meaningful. ${ }^{22}$ It was estimated there will be a SD of 16.3 in the VAS scores based on previous research on a similar participant population. ${ }^{23}$ Accordingly, assuming an $\alpha$ of $5 \%$ and a $\beta$ error level of $10 \%, 40$ participants were required within each footwear group.

Survival analysis was carried out using each injury as an event. Once a participant had registered an injury event they were excluded from further survival analysis. The Kaplan-Meier 
model is then based on estimating conditional probabilities at each time point when an event occurs and taking the product limit of those probabilities to estimate the survival rate at each point in time. Post hoc analysis of survival data was performed with a log rank test for comparing the equality of survival distributions where all time points are weighted equally in this test.

A Shapiro-Wilks test of normality preceded a repeated measures analysis of variance (RMANOVA) that determined whether there was a significant main effect for footwear from baseline to 12 weeks for the FADI and VAS scores. A pairwise comparison across groups was then performed at the baseline and 12-week time points within the RMANOVA model. Based on their potential to influence injury rates in previous studies, the following variables were included in the model as covariates: age, body mass index, severity of prior history of running injury, Hallux ROM, ankle ROM, Q-angle and prior proportion of weekly time running on road surfaces.

Injury incidence will be calculated as the number of injury events divided by athletic exposures (each running session is an athletic exposure), and then normalised to represent the incidence rate per 1000 athletic exposures. The absolute risk reduction and relative risk for either the full or partial minimalist shoe will be determined by the equations below:

$$
\begin{gathered}
\mathrm{ARR}=\mathrm{P}_{\text {inj(con })}-\mathrm{P}_{\text {inj(min) }} \\
\mathrm{RR}=\frac{\mathrm{P}_{\text {inj(min) }}}{\mathrm{P}_{\text {inj(con })}}
\end{gathered}
$$

where ARR is absolute risk reduction, $\mathrm{RR}$ relative risk, $\mathrm{P}_{\text {inj(min) }}$ the injury rate as a per cent wearing either the partial or full minimalist shoe, $\mathrm{P}_{\text {inj(con) }}$ injury rate as a per cent wearing the conventional neutral support running shoe.

The $\alpha$ for all statistical tests performed in this study is set to 0.05 .

\section{RESULTS}

One hundred and three participants were enrolled in this study from a total of 167 screened individuals. Participants were recruited largely from newspaper advertisements and word-of-mouth. One hundred and three individuals took part in the baseline assessment; however, four individuals dropped out prior to starting the programme and before wearing their assigned footwear (two thought it was too great a time commitment (one neutral, one partial minimalist), one had a cycling accident fracturing their pelvis (partial minimalist) and one had a running injury between baseline testing and starting to use their assigned footwear (neutral); figure 1). There were an additional eight participants lost to follow-up (one with knee injury from kick-boxing (partial minimalist), five unable to commit to programme $(2 \times$ partial minimalist, $1 \times$ neutral, $2 \times$ full minimalist), one with ankle injury of unknown cause (partial minimalist) and one from ice-hockey injury (full minimalist)) subsequent to the baseline appointment whose data were included in the analysis.

Overall compliance to footwear usage was very good, ranging from $96 \%$ to $97 \%$ (no significant group effect). Compliance to the running programme averaged $66.6 \%$ of assigned workouts being completed (ranging from 59\% to 75\%across groups, with no significant group effect). Sixty-nine participants (70\%) completed the $10 \mathrm{~K}$ event at the conclusion of the programme with 31 runners posting a new personal best time $35 \%$ within NF, 24\% within PMF and 31\% within FMF groups) (differences not-significant). There were no significant differences in any of our independent variables at baseline (see online supplementary table S2).

Overall there were 23 injury events recorded over the 12 -week period resulting in an injury rate of $23.2 \%$ (see online supplementary table S3). There were more injuries in both minimalist groups than the neutral group contributing to a $160 \%$ and $310 \%$ RR of injury in the full minimalist and partial minimalist group, respectively. Kaplan-Meier analysis reported a significant difference in the number of injury events over time across footwear condition driven by the disproportionate number of injury events reported by runners in the partial minimalist group (figure 2). Based on injury event data, there is a higher likelihood of experiencing an injury with minimalist footwear, particularly with the partial minimalist condition.

Outcomes from the RMANOVA report no significant group effect for FADI scores (figure 3).

For the VAS pain scales, comparison of the pain scores over the 12-week period report little difference (see online supplementary table S4). Only in VAS for shin/calf pain did participants wearing full minimalist footwear report significantly $(p<0.01)$ greater pain than both other footwear groups (see online supplementary table S5).

\section{DISCUSSION}

This study is the first to longitudinally examine the epidemiological effects of two different models of minimalist footwear. The participants in this study were recreational habitual runners without previous experience running barefoot or with other minimalist footwear models preparing for a $10 \mathrm{~km}$ event. The overall injury rate of $23.2 \%$ is low compared with other similar exposure times to running in the literature that speculatively may be a function of the running programme or our selection criteria (ie, being injury-free for the preceding 6 months). ${ }^{24} 25$

On the basis of clinical data from this study our hypotheses were supported. The first hypothesis was supported with respect to minimalist footwear modifying injury risk. Injury event analysis showed there was a higher likelihood of experiencing an injury with minimalist footwear compared with a conventional neutral shoe model, with the partial minimalist condition having a particularly higher risk. The outcomes from the overall VAS for pain and FADI scores support our second hypothesis. Both minimalist shoes resulted in changes to reported pain and foot/ankle function at the shin/calf, knee, hip and lower back.

Compared with conventional neutral-supported shoes, wearing minimalist footwear appears to place the unfamiliar runner at an overall greater risk of experiencing an injury that stops running or experiencing running-related pain. This apparent injury risk was not equal across the minimalist conditions. Runners in the partial minimalist condition experienced threetimes as many injury events as the neutral group, and five more injuries than the FMF group. Runners in the full minimalist condition reported greater shin and calf pain.

There is limited, and conflicting, experimental data on the biomechanical effects of wearing partial minimalist running footwear. Previously, it has been shown that use of a similar model of Nike Free (V.5.0) for routine activities three times per week modify neuromotor activation and increase muscle crosssectional area for the long and intrinsic toe flexor group. ${ }^{12}$ The therapeutic effect of the Nike Free V.5.0 for treating chronic plantar fasciopathy may have been a result of such neuromuscular changes. ${ }^{26}$ Specifically use of the Nike Free V.5.0 could have resulted in an increase in foot flexor strength secondary to the greater flexibility of this shoe's forefoot. ${ }^{27}$ In contrast, a recent biomechanical analysis of the same model of Nike Free used in 


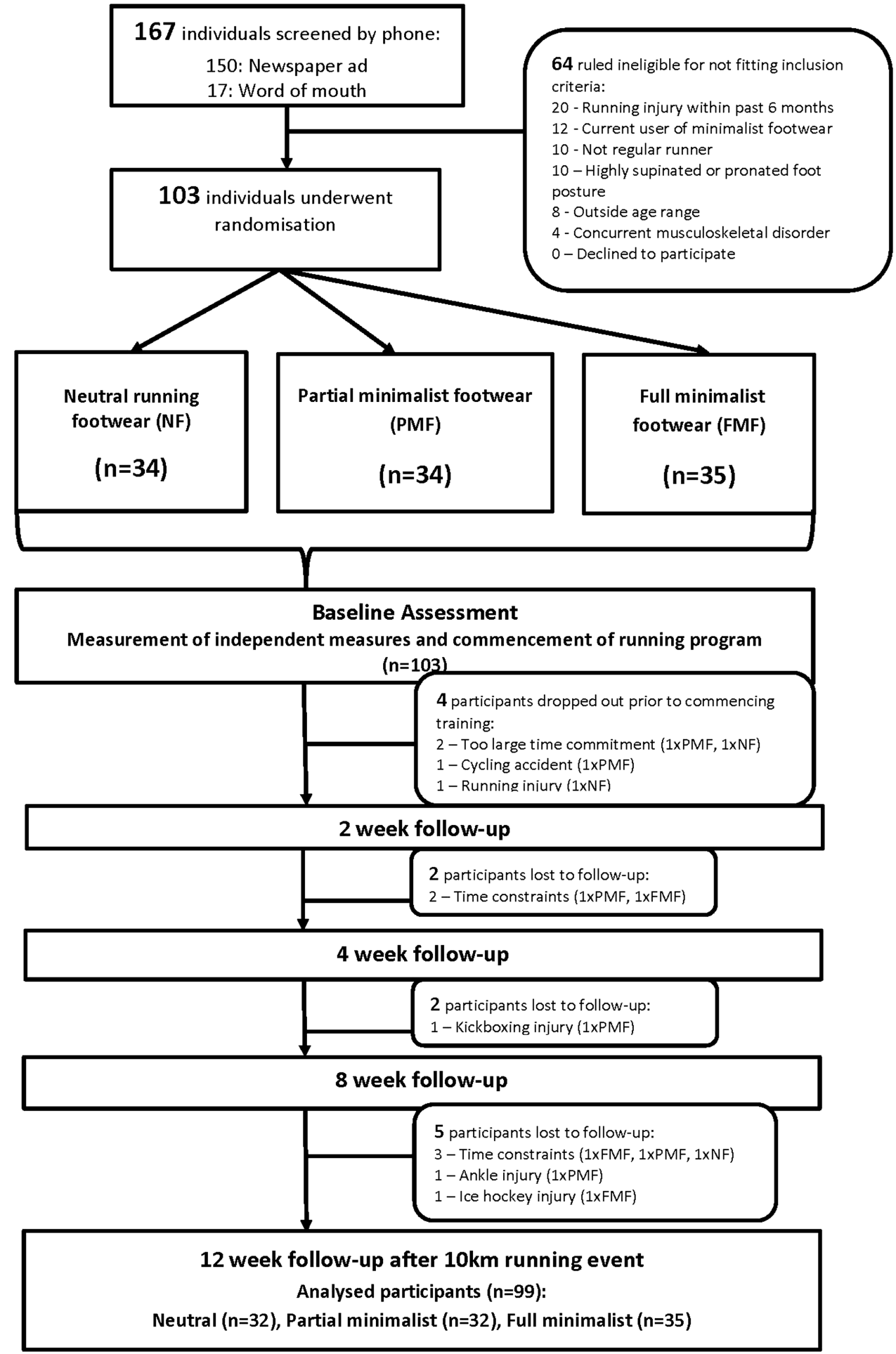

Figure 1 Participant flow through screening, randomisation, training protocol and follow-up in this study. Participants are considered 'drop-outs' if they were enrolled and randomised but did not participate in the training programme or complete a follow-up assessment. Participants 'lost to follow-up' were included in the final analysis and had missing data only after completing a 2-week outcome assessment. Numbers lost to follow-up are indicated at their respective time point. FMF, full minimalist footwear; NF, neutral footwear; PMF, partial minimalist footwear.

the present study (V.3.0) did not report any substantial difference in biomechanics between the Nike Free compared to otherwise conventional running footwear. ${ }^{28}$ Considering the effect of the Nike Free is thought to have a sizable effect on intrinsic foot motion and strength, we encourage future studies on this shoe (and other minimalist designs) to examine this footwear category's effect on rearfoot-forefoot motion and dynamics. We speculate that the larger injury rate in the group wearing the Nike Free, in comparison to the group wearing the 5-Fingers, may indeed be a function of the partial reduction of the Nike Free's sole. By only partially reducing midsole materials, the Nike Free might compromise the shock attenuation and stability 


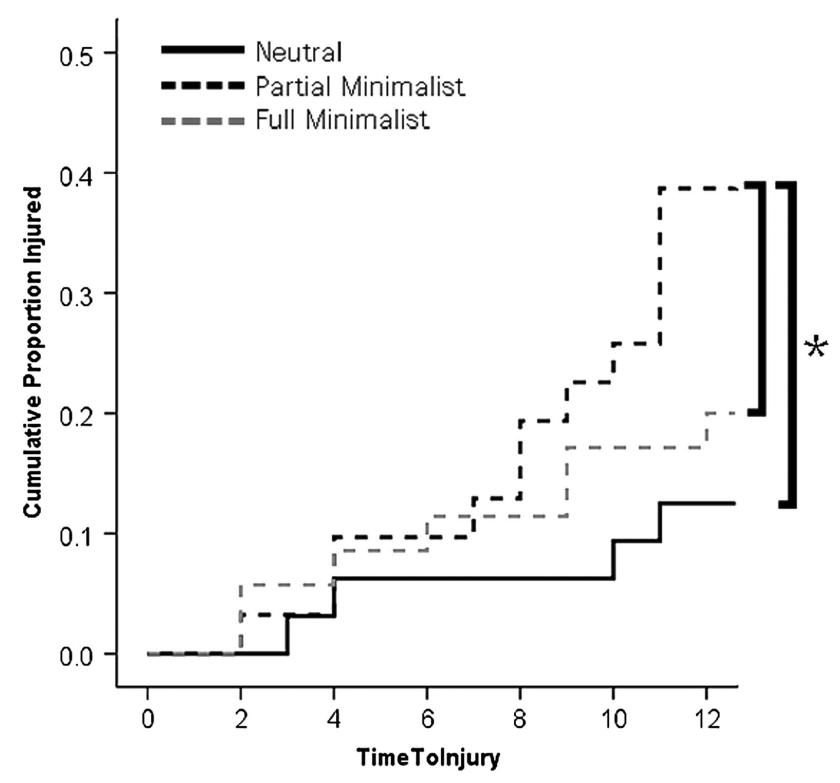

Figure 2 Kaplan-Meier plot of survival analysis illustrating the time sequence of injury for runners in the three footwear groups. *Indicates a significant difference across groups at $\mathrm{p}<0.05$.

properties of the full neutral shoe, yet maintain enough cushion to blunt the senosory-motor drive to modify gait possibly seen in runners wearing the 5-Fingers. ${ }^{3}$ Importantly, no study has longitudinally assessed the biomechanical and neuromuscular effects of persistent minimalist footwear use; therefore, it remains difficult to account mechanistically for the injury rates across footwear conditions seen in the present study.

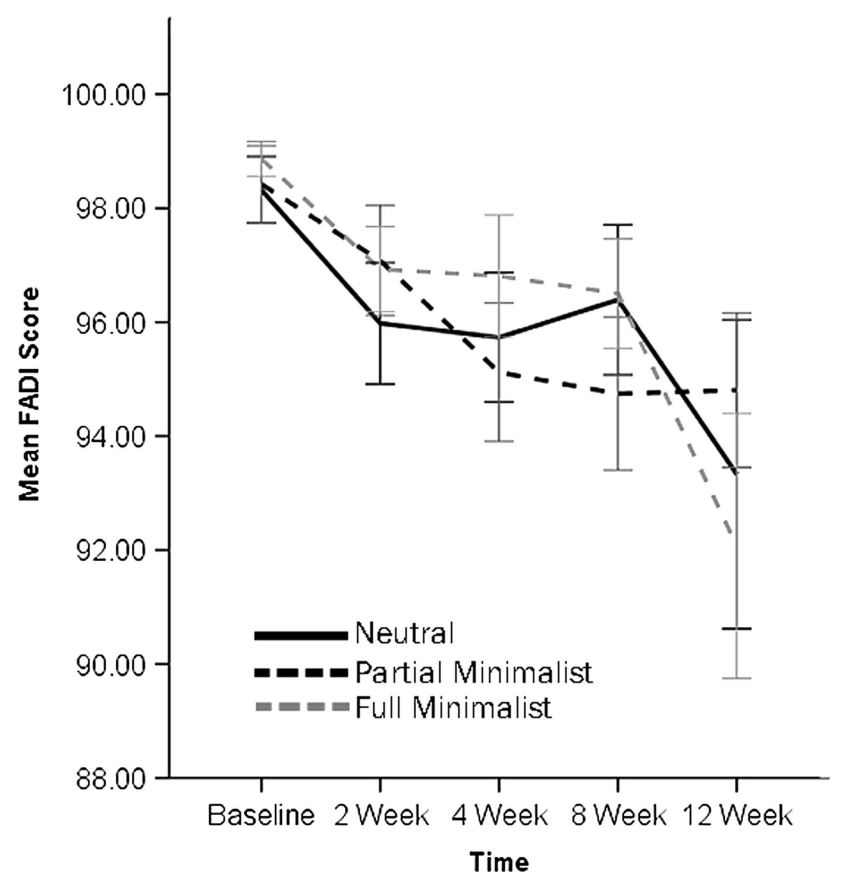

Figure 3 Foot and ankle disability index scores across the three footwear conditions. Bars indicate SE. FMF, full minimalist footwear; NF, neutral footwear; PMF, partial minimalist footwear.
It is noteworthy that runners in full minimalist footwear condition reported greater calf and shin pain throughout the 12 -week period. This finding was not unexpected given the likelihood that some of the runners in the full minimalist footwear condition adopted a forefoot strike pattern that could have resulted in greater (and unaccustomed) loading of the Achilles tendon and triceps surae musculature secondary to a larger ankle dorsiflexion moment immediately following touchdown. ${ }^{3}$ The greater heel height in the partial minimalist footwear likely mitigated this loading on the shank. Preliminary clinical evidence suggests that runners may experience an increased risk of stress fractures to the foot and injury to the plantar fascia after transitioning from conventional running footwear to a full minimalist footwear condition, yet there was no increase in foot pain reported in the participants in the full minimalist footwear group. ${ }^{29}{ }^{30}$ It is advisable that an appropriate period of transition to a minimalist footwear category based on individualised criteria should be established for each runner. Currently, however, there is no consensus on how such a progression should be made.

\section{Strengths and limitations}

There are important limitations when interpreting outcomes from the present study. A diagnosis for the participants who did report injuries was not available and as a result there is some loss in the precision and accuracy of the injury data in this study. However, validated outcome measures for assessing pain, as well as ankle and foot disability were administered concurrent to the injury status for thorough reporting of a runner's 'clinical status'. The observation period in this study is relatively short at 12 weeks; the long-term clinical effects of habitual minimalist footwear use remain unknown. Participants were not blinded to their group allocation, a process that would be difficult to achieve in the context of studies on footwear minimalism. Additional data regarding concurrent sport participation was not recorded and may have influenced the outcomes. Strengths of this study are a random allocation of footwear condition (including the control or neutral footwear condition), similar training exposure, good participant study adherence and denominator-based reporting of injury incidence.

In conclusion, running in minimalist footwear appears to increase the likelihood of experiencing an injury and running-related pain in runners otherwise new to this footwear category while training for a $10 \mathrm{~km}$ event. Furthermore, it remains unknown to what extent, if any, a preparatory strengthening or conditioning programme would have at mitigating the injury risk associated with minimalist footwear. It is recommended that clinicians exercise caution when recommending minimalist footwear to runners without prior experience using this type of footwear.

\section{What are the new findings?}

- Both partial and full minimalist footwear designs resulted in a greater risk of injury compared with the neutral footwear group.

- The partial minimalist shoe resulted in a greater overall injury rate.

- Runners in the full minimalist shoe reported greater shin and calf pain than runners in both other footwear groups. 


\section{How might it impact clinical practice in the near future?}

- It is recommended that clinicians use caution when prescribing the use of minimalist running shoe designs in light of the greater risk of injury and pain with their use.

- This study reports the effects of novel minimalist footwear usage for running; it remains unknown to what extent, if any, a preparatory strengthening or conditioning programme would have at mitigating the injury risk associated with minimalist footwear.

Acknowledgements The authors wish to thank Phil Moore, and his retail store Lady Sport, for their valued expertise and assistance in coordinating the running clinics used in this study. The following volunteers helped with data collection and participant management without which this study would not be possible: Jamie Hartwell, Duncan McGuinty, Stephanie Hay and Brent Day.

Contributors MR planned and assisted with conducting and reporting of the study. ME conducted the study. RN-W assisted with reporting of the study. JT assisted with planning and reporting of the study. MR and JT are nominated as guarantors for the content.

Funding This study was funded through an industry partnership grant with Nike Inc. Jack Taunton received funding to his institution to support the research reported in this manuscript. Michael Ryan and Maha Elashi received consulting payments for work to carry-out this research.

\section{Competing interests None.}

Ethics approval Clinical Research Ethics Board of the Univeristy of British Columbia.

Provenance and peer review Not commissioned; externally peer reviewed.

\section{REFERENCES}

1 Bramble DM, Lieberman DE. Endurance running and the evolution of Homo. Nature 2004;432:345-52.

2 Lieberman DE, Bramble DM. The evolution of marathon running: capabilities in humans. Sports Med 2007;37:288-90.

3 Lieberman DE, Venkadesan M, Werbel WA, et al. Foot strike patterns and collision forces in habitually barefoot versus shod runners. Nature 2010;463:531-5.

4 Robbins SE, Hanna AM. Running-related injury prevention through barefoot adaptations. Med Sci Sports Exerc 1987;19:148-56.

5 De Wit B, De Clercq D, Aerts P. Biomechanical analysis of the stance phase during barefoot and shod running. J Biomech 2000;33:269-78.

6 Divert $\mathrm{C}$, Mornieux G, Baur $\mathrm{H}$, et al. Mechanical comparison of barefoot and shod running. Int J Sports Med 2005;26:593-8.

7 Squadrone R, Gallozzi C. Biomechanical and physiological comparison of barefoot and two shod conditions in experienced barefoot runners. I Sports Med Phys Fitness 2009;49:6-13.

8 Derrick TR, Hamill J, Caldwell GE. Energy absorption of impacts during running at various stride lengths. Med Sci Sports Exerc 1998;30:128-35.
9 Heiderscheit BC, Chumanov ES, Michalski MP, et al. Effects of step rate manipulation on joint mechanics during running. Med Sci Sports Exerc 2011:43:296-302.

10 Rixe JA, Gallo RA, Silvis ML. The barefoot debate: can minimalist shoes reduce running-related injuries? Curr Sports Med Rep 2012;11:160-5.

11 Noakes T. Lore of running. 4th edn. Champaign, IL: Oxford University Press, 2001.

12 Bruggemann $G$, Potthast W, Braunstein B, et al. Effect of increased mechanical stimuli on foot muscles functional capacity. ISB. Cleveland: American Society of Biomechanics, 2005:553.

13 Redmon A, Crosbie J, Ouvrier R. Development and validation of a novel rating system for scoring standing foot posture: the foot posture index. Clin Biomech 2006;21:89-98.

14 Redmond A, Crosbie J, Ouvrier R. Development and validation of a novel rating system for scoring standing foot posture: the foot posture index. Clin Biomech (Bristol, Avon) 2006;21:89-98.

15 Keenan A, Redmon A, Horton $M$, et al. The foot posture index: rasch analysis of a novel, foot-specific outcome measure. Arch Phys Med Rehabil 2007;88:88-93.

16 Redmon A, Crane Y, Menz H. Normative values for the foot posture index. J Foot Ankle Res 2008;1:6.

17 Munuera PV, Trujillo P, Guiza I. Hallux interphalangeal joint range of motion in feet with and without limited first metatarsophalangeal joint dorsiflexion. J Am Podiatr Med Assoc 2012;102:47-53.

18 Rauh MJ, Koepsell TD, Rivara FP, et al. Quadriceps angle and risk of injury among high school cross-country runners. J Orthop Sports Phys Ther 2007;37:725-33.

19 Wen DY, Puffer JC, Schmalzried TP. Injuries in runners: a prospective study of alignment. Clin J Sport Med 1998;8:187-94.

20 Pledger G. Compliance in clinical trials: impact on design, analysis and interpretation. In: Schmidt D, Leppik I, eds. Compliance in epilepsy. Elsevier Science Publishers, 1988:125-33.

21 Friedman L, Furberg C, DeMets D. Fundamentals of clinical trials. 3rd edn. New York, NY: Springer, 1998.

22 Tashjian R, Deloach J, Porucznik C, et al. Minimal clinically important differences (MCID) and patient acceptable symptomatic state (PASS) for visual analog scales (VAS) measuring pain in patients treated for rotator cuff disease. J Shoulder Elbow Surg 2009;18:927-32.

23 Ryan MB, Valiant GA, McDonald K, et al. The effect of three different levels of footwear stability on pain outcomes in women runners: a randomised control trial. Br J Sports Med 2011;45:715-21.

24 Taunton JE, Ryan MB, Clement DB, et al. A prospective study of running injuries: the Vancouver Sun Run 'In Training' clinics. Br J Sports Med 2003;37: 239-44.

25 van Gent RN, Siem D, van Middelkoop M, et al. Incidence and determinants of lower extremity running injuries in long distance runners: a systematic review. $\mathrm{Br}$ J Sports Med 2007;41:469-80; discussion 80.

26 Ryan $M$, Fraser $S, M c D o n a l d ~ K$, et al. Examining the degree of pain reduction using a multielement exercise model with a conventional training shoe versus an ultraflexible training shoe for treating plantar fasciitis. Phys Sportsmed 2009;37:68-74.

27 Nike Free Running Study. Proceedings of the 7th Conference of the International Sport Engineering Association; 2008; Biarritz, France.

28 Bonacci J, Saunders PU, Hicks A, et al. Running in a minimalist and lightweight shoe is not the same as running barefoot: a biomechanical study. Br J Sports Med 2013;47;387-392.

29 Giuliani J, Masini B, Alitz C, et al. Barefoot-simulating footwear associated with metatarsal stress injury in 2 runners. Orthopedics 2011;34:e320-3.

30 Salzler MJ, Bluman EM, Noonan S, et al. Injuries observed in minimalist runners. Foot Ankle Int 2012;33:262-6. 


\section{BISM Examining injury risk and pain perception in runners using minimalist footwear}

Michael Ryan, Maha Elashi, Richard Newsham-West, et al.

Br J Sports Med published online December 19, 2013

doi: 10.1136/bjsports-2012-092061

Updated information and services can be found at:

http://bjsm.bmj.com/content/early/2013/12/19/bjsports-2012-092061.full.html

\section{These include:}

\section{Data Supplement "Supplementary Data"}

http://bjsm.bmj.com/content/suppl/2013/12/19/bjsports-2012-092061.DC1.html

References This article cites 25 articles, 6 of which can be accessed free at: http://bjsm.bmj.com/content/early/2013/12/19/bjsports-2012-092061.full.html\#ref-list-1

$\mathbf{P}<\mathbf{P} \quad$ Published online December 19, 2013 in advance of the print journal.

Email alerting

Receive free email alerts when new articles cite this article. Sign up in service the box at the top right corner of the online article.

Notes

Advance online articles have been peer reviewed, accepted for publication, edited and typeset, but have not not yet appeared in the paper journal. Advance online articles are citable and establish publication priority; they are indexed by PubMed from initial publication. Citations to Advance online articles must include the digital object identifier (DOIs) and date of initial publication.

To request permissions go to:

http://group.bmj.com/group/rights-licensing/permissions

To order reprints go to:

http://journals.bmj.com/cgi/reprintform

To subscribe to BMJ go to:

http://group.bmj.com/subscribe/ 